\title{
The neurological underpinnings of cluttering: some initial findings
}

Article

Accepted Version

Ward, D., Connally, E. L., Pliatsikas, C., Bretherton-Furness, J. and Watkins, K. E. (2015) The neurological underpinnings of cluttering: some initial findings. Journal of Fluency Disorders, 43. pp. 1-16. ISSN 0094-730X doi:

https://doi.org/10.1016/j.jfludis.2014.12.003 Available at https://centaur.reading.ac.uk/39265/

It is advisable to refer to the publisher's version if you intend to cite from the work. See Guidance on citing.

To link to this article DOI: http://dx.doi.org/10.1016/j.jfludis.2014.12.003

Publisher: Elsevier

All outputs in CentAUR are protected by Intellectual Property Rights law, including copyright law. Copyright and IPR is retained by the creators or other copyright holders. Terms and conditions for use of this material are defined in the End User Agreement.

\section{www.reading.ac.uk/centaur}

\section{CentAUR}

Central Archive at the University of Reading 
Reading's research outputs online 


\title{
The neurological underpinnings of cluttering: Some initial findings
}

\author{
David Ward $^{\mathrm{a}, \mathrm{b}, *}$, Emily L. Connally ${ }^{\mathrm{c}}$, Christos Pliatsikas ${ }^{\mathrm{d}}$, \\ Jess Bretherton-Furness ${ }^{a}$, Kate E. Watkins ${ }^{c}$ \\ a School of Psychology and Clinical Language Sciences, The University of Reading, Reading RG6 6AA, UK \\ ${ }^{\mathrm{b}}$ Apple House Centre for Stammering, Warneford Hospital, Oxford OX3 7JX, UK \\ c Department of Experimental Psychology, South Parks Road, University of Oxford, Oxford, OX1 3UD, UK \\ d Centre for Integrative Neuroscience and Neurodynamics, The University of Reading, Reading RG6 6AA, UK
}

\section{A R T I C L E I N F O}

\section{Article history:}

Received 8 August 2013

Received in revised form

15 December 2014

Accepted 28 December 2014

Available online $\mathrm{xxx}$

\section{Keywords:}

Fluency

Cluttering

Stuttering

fMRI

\begin{abstract}
A B S T R A C T
Background: Cluttering is a fluency disorder characterised by overly rapid or jerky speech patterns that compromise intelligibility. The neural correlates of cluttering are unknown but theoretical accounts implicate the basal ganglia and medial prefrontal cortex. Dysfunction in these brain areas would be consistent with difficulties in selection and control of speech motor programs that are characteristic of speech disfluencies in cluttering. There is a surprising lack of investigation into this disorder using modern imaging techniques. Here, we used functional MRI to investigate the neural correlates of cluttering.

Method: We scanned 17 adults who clutter and 17 normally fluent control speakers matched for age and sex. Brain activity was recorded using sparse-sampling functional MRI while participants viewed scenes and either (i) produced overt speech describing the scene or (ii) read out loud a sentence provided that described the scene. Speech was recorded and analysed off line. Differences in brain activity for each condition compared to a silent resting baseline and between conditions were analysed for each group separately (cluster-forming threshold $Z>3.1$, extent $p<0.05$, corrected) and then these differences were further compared between the two groups (voxel threshold $p<0.01$, extent $>30$ voxels, uncorrected).

Results: In both conditions, the patterns of activation in adults who clutter and control speakers were strikingly similar, particularly at the cortical level. Direct group comparisons revealed greater activity in adults who clutter compared to control speakers in the lateral premotor cortex bilaterally and, as predicted, on the medial surface (pre-supplementary motor area). Subcortically, adults who clutter showed greater activity than control speakers in the basal ganglia. Specifically, the caudate nucleus and putamen were overactive in adults who clutter for the comparison of picture description with sentence reading. In addition, adults who clutter had reduced activity relative to control speakers in the lateral anterior cerebellum bilaterally.

Eleven of the 17 adults who clutter also stuttered. This comorbid diagnosis of stuttering was found to contribute to the abnormal overactivity seen in the group of adults who clutter in the right ventral premotor cortex and right anterior cingulate cortex. In the remaining areas of abnormal activity seen in adults who clutter compared to controls, the subgroup who clutter and stutter did not differ from the subgroup who clutter but do not stutter.
\end{abstract}

* Corresponding author at: Corresponding author at: School of Psychology and Clinical Language Sciences, The University of Reading, Reading RG6 6AA, UK. Tel.: +44011378 4689 .

E-mail address: d.ward@reading.ac.uk (D. Ward). 
Conclusions: Our findings were in good agreement with theoretical predictions regarding the neural correlates of cluttering. We found evidence for abnormal function in the basal ganglia and their cortical output target, the medial prefrontal cortex. The findings are discussed in relation to models of cluttering that point to problems with motor control of speech.

Educational objectives: This paper reports findings on the neural correlates seen in adults who clutter, and offers hypotheses as to how these might map onto the behaviours seen amongst those who clutter. Readers will be able to (a) identify the structures that are implicated in the disorder of cluttering, (b) understand arguments relating these structures to the behavioural expression of the disorder, (c) understand some of the complexities in interpreting data pertaining to recovery from cluttering, (d) understand where future efforts in research into the neurological correlates of cluttering should be focussed.

(c) 2015 Published by Elsevier Inc.

\section{Introduction}

\subsection{A unified perspective of cluttering}

Cluttering has been recognised and described as a communication disorder arguably as far back as stuttering (Van Riper, 1982), but rigorous scientific examination of the disorder has until very recently remained limited. The difficulty in some part has involved a certain circularity to the problem of identifying the significant features of cluttering. There is also a history of dispute as to the scope of the core behaviours of the disorder, with speculation as to whether cluttering is a motor speech disorder, a language disorder, both, or perhaps more to do with executive functioning than either (Daly, 1986; Preus, 1996; St. Louis, Myers, Bakker, \& Raphael, 2007; Van Zaalen, Wijnen, \& De Jonckere, 2009a; Ward, 2010; Weiss, 1964). These differences have led researchers to apply different criteria when defining their experimental groups. It has been helpful, then, that St. Louis and Schulte (2011) recently refined their working definition of cluttering, in what they call the 'lowest common denominator' (LCD) definition. As the name implies, this is conservative perspective, including only a limited number of core cluttering characteristics. They acknowledge that this definition may subsequently need to be revised and updated as more is known about cluttering, but it provides what is currently taken by clinicians and researchers alike as the standard definition of the disorder:

Cluttering is a fluency disorder wherein segments of conversation in the speaker's native language typically are perceived as too fast overall, too irregular, or both. The segments of rapid and/or irregular speech rate must further be accompanied by one or more of the following: (a) excessive 'normal' disfluencies; (b) excessive collapsing or deletion of syllables; and/or (c) abnormal pauses, syllable stress, or speech rhythm (St. Louis E Schulte, 2011, pp. 241-242).

St. Louis and Schulte (2011) qualify this definition further as follows: for example, that cluttering need not occur frequently but sufficiently often to exceed that seen in normal speakers; that the irregular speech rate may be described as "jerky" or "spurty"; and, that collapsing of syllables can include excessive shortening, "telescoping," or "over-coarticulating" various syllables, especially in multisyllabic words.

\subsection{Recent considerations on the aetiology of cluttering}

Cluttering behaviour, even when constrained under the LCD definition, might still be caused by a wide range of factors. For example, the hesitations and normal non-fluencies cited in the LCD could be language based, motor based, or reflect a more generalised problem with organisation, or planning as Weiss (1964) first speculated. Ward (2011a) argued that cluttering symptoms are seen at all levels of Van der Merwe's (2008) four-level model of speech processing. This starts with linguistic planning levels, but then involves motor planning, motor programming, and finally, motor execution. Relatedly, Ward (2006, 2011a, 2011b) identified two possible subcomponents of cluttering: those which affect what he termed 'motoric fluency', relating to aspects such as overcoarticulation, and those which impact on 'linguistic fluency', for example filled pauses, part word repetitions, unnatural pausing and phrase revisions. Recently, Van Zaalen, Wijnen, and De Jonkere (2009a, 2009b) have argued that, unlike stuttering, cluttering is a language-based disorder. In their view, the disorder can be split into two subtypes: 'syntactic cluttering', which somewhat equates to Ward's concept of linguistic cluttering, and 'phonemic cluttering' which covers areas Ward considered to be motoric. It is possible that an investigation into the brain and the functional abnormalities associated with cluttering may go some way to helping resolve these fundamental questions.

\subsection{The neural basis of cluttering}

Very little is known about the neural basis of cluttering. Earlier speculation aligned the disorder with deficits in motor speech control, with Seeman (1970) suggesting that cluttering results from a disturbance of the basal ganglia circuitry. 
Lebrun (1996) observed that traits of cluttering after brain damage or disease typically occur after damage to the basal ganglia system, as in Parkinson's disease (PD).

The potential association of cluttering with dysfunction in basal ganglia processing is one that has recently been elucidated in a detailed examination of possible neural correlates. Alm (2010) suggested that stuttering might be related to signal inhibition within the basal ganglia, which results in the blocks and mistimings that are core to the disorder, and that cluttering might result from disinhibition, or premature release of the signals, within the same circuitry. Alm (2011) later integrated this possibility into a model which also implicated cortical structures. He speculated that a number of sites might be involved, but that the core of cluttering lay in the medial wall of the left frontal lobe. This 'executive hub' model holds that the medial frontal cortex operates in parallel with traditional language-processing centres in the left hemisphere, such as Broca's and Wernicke's areas. Alm (2011) further argues that the heterogeneous speech behaviours seen in cluttering are likely to implicate different neural mechanisms and pathways for different subgroups, and this seems consistent with Ward's (2010, 2011b) concept of cluttering as a spectrum disorder.

\subsection{Aims}

As noted above, there is a dearth of evidence regarding the brain structure and functional abnormalities associated with a diagnosis of cluttering. We know of no previous reports that used imaging to investigate brain function in people who clutter compared to people who are normally fluent. The aim of the current study was - in the first instance - to address this gap in the literature. We scanned a large group of adults who clutter $(N=17)$, the majority of which $(N=11)$ also had a comorbid diagnosis of stuttering. We compared them to a group of control speakers matched for age, sex and handedness. We used two conditions during which participants spoke out loud in the scanner: picture description and sentence reading. Compared to sentence reading, using one's own speech in the picture description condition is a more complex task in terms of motor planning and also places greater demands on linguistic planning, (e.g. selection of lexical items and construction of the syntactic frame). Implementation of these two conditions allowed us, therefore, to address the second aim of our study, which was to better assess the relevance of language processing and motor speech components for people who clutter.

\section{Methods}

\subsection{Participants}

Seventeen adults who clutter (13 males, 4 females; aged 20-55 years, 4 left-handers) and 17 age- and sex-matched controls (13 males, 4 females; aged 19-53 years; 3 left-handers) were scanned using functional MRI (see Table 1). Participants were recruited through the clinical contacts of the first author (DW), and through advertisement on the British Stammering Association website. All adults who clutter had been diagnosed as cluttering by a qualified speech and language therapist (DW) using the LCD definition of cluttering (St. Louis \& Schulte, 2011). Recall that this definition implicates a speech rate that appears to be overly rapid or jerky in delivery. At least one of three additional features must also be present: excessive number of nonstuttering like dysfluencies, abnormal pausing or speech rhythm, excessive coarticulation (see Section 1.1 for the complete definition).

Eleven of the adults who clutter had comorbid stuttering ranging in severity from very mild to mild as assessed by a speech language therapist (DW) using the Stuttering Severity Instrument-3 (Riley, 1994). No psychological testing or attentional assessments were administered. No testing for dyspraxia, dyslexia, or autism spectrum disorder was undertaken, but participants were asked if they had ever been diagnosed with any of these disorders. Of the 11 adults who cluttered and stuttered, one reported a diagnosis of developmental dyspraxia and another had a history of dyslexia. Of the six adults who clutter who did not stutter, one reported a diagnosis of developmental dyspraxia, and another of developmental dyspraxia and dyslexia. A third had a diagnosis of autism spectrum disorder. Consistent with the diagnosis of a developmental (nonspeech) dyspraxia, no participants showed signs of verbal dyspraxia in conversation, and none had ever sought or required speech language therapy for these conditions. No controls had a history or diagnosis of learning or other speech and language disorders.

All participants gave informed consent to their participation in the research, and the project received approval from the University of Reading's ethics committee.

Table 1

Group demographics.

\begin{tabular}{lrccc}
\hline Group & $N(\mathrm{M}: \mathrm{F})$ & Left-handed (M:F) & Mean age (years) & Age range (years) \\
\hline Controls & $17(13: 4)$ & $3(2: 1)$ & 32.4 & $19-53$ \\
AWC and do not stutter $^{\mathrm{a}}$ & $6(5: 1)$ & 0 & 33.5 & $23-55$ \\
AWC and stutter $^{\mathrm{b}}$ & $11(8: 3)$ & $4(3: 1)$ & 34.3 & $20-55$ \\
\hline
\end{tabular}

AWC, adults who clutter; M, male; F, female.

a One participant also had dyspraxia, one had dyspraxia and dyslexia, one had a diagnosis of autism spectrum disorder

b One participant also had dyspraxia, one had a history of dyslexia. 


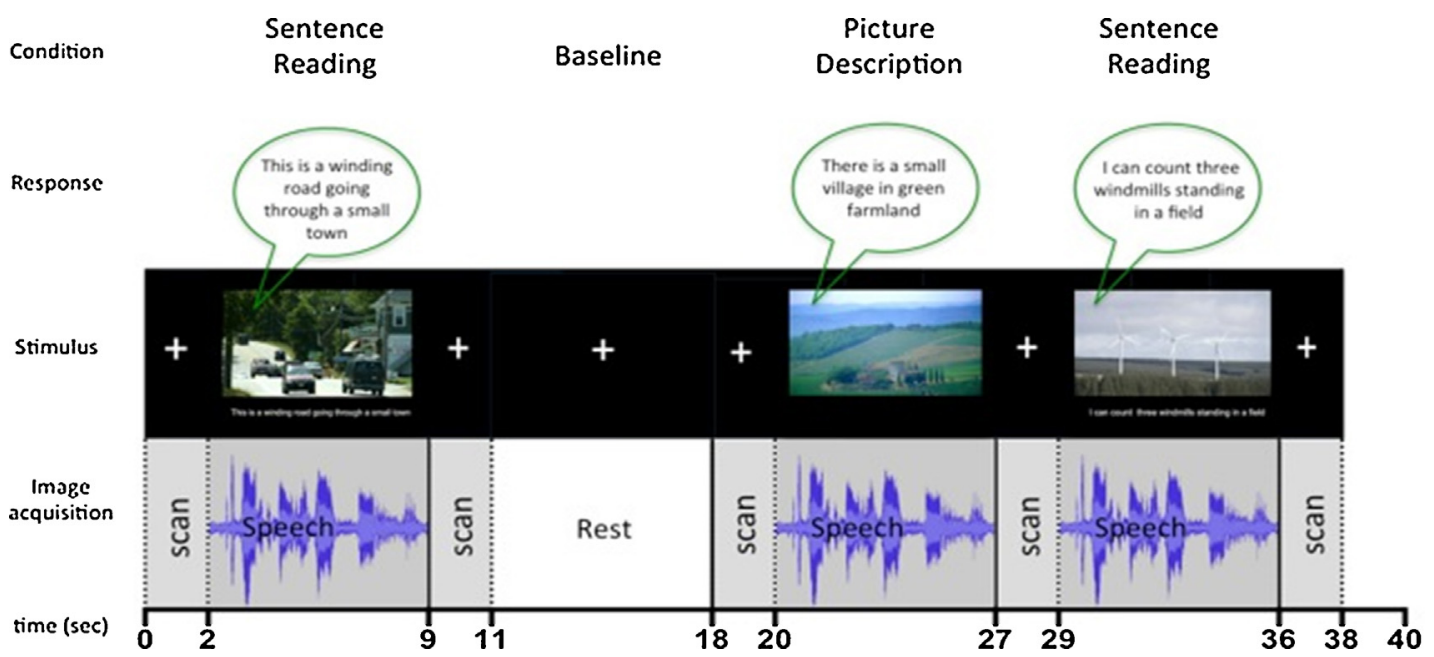

Fig. 1. Task, design and scanner acquisition method. Two conditions sentence reading and picture description were used to assess speech-related brain activity. Sparse sampling was used to measure the MRI signal evoked by speech production during the 7-s gaps between scans.

\subsection{Data acquisition}

MRI data were obtained using The University of Reading's 3-T Siemens MAGNETOM Trio MRI scanner with Syngo software and a 12-channel head coil. A T1-weighted high-resolution scan of brain anatomy was acquired using an MPRAGE sequence with $1 \mathrm{~mm}^{3}$ voxels $\left(T R=2020 \mathrm{~ms}, \mathrm{TE}=2.9 \mathrm{~ms}\right.$, flip angle $=9^{\circ}$ ). Two functional MRI scan runs were obtained in each participant which each comprised 120 whole-head T2*-weighted echo-planar images (TE $=30 \mathrm{~ms}$ ). Within these runs, image volumes were acquired every $9 \mathrm{~s}$ with a silent delay of $7 \mathrm{~s}$ (i.e. sparse sampling) between volumes. Each volume comprised 2 -s acquisition of $324-\mathrm{mm}$ thick axial slices (in-plane resolution $3 \mathrm{~mm} \times 3 \mathrm{~mm}$ ). During the 7-s silent delay between measurements, subjects saw a stimulus via scanner-compatible goggles (NordicNeuroLab Visual System, SVGA, resolution: 800 $(3 \times) \times 600,16.7$ million colours, refresh rate: $75 \mathrm{~Hz}$, field of view: $30^{\circ}$ horizontal, $23^{\circ}$ vertical) that was either a picture with a descriptive sentence (Sentence Reading condition), a picture with no text (Picture Description condition), or a blank screen (Baseline). A '+' appeared in the middle of the screen during the 2-s acquisition (see Fig. 1).

\subsection{Procedure}

Prior to the scan, the experimental task was explained to the participants and they had the opportunity to ask questions. They were instructed to read the sentences aloud whenever a sentence accompanied the picture. These sentences were related to the pictures shown (see Fig. 1). In the picture description condition, participants were told to produce a sentence overtly and that they could describe the picture if they wished or say anything related or unrelated to it. Participants were told to remain silent when they saw a blank screen and to stop speaking when the crosshair appeared so that there would be no speech-related movement of the head during data acquisition. Following the instructions, participants undertook a 3-min practice with stimuli not subsequently used in the scanner. This demonstrated how the stimuli would appear to them once in the scanner, the rate of presentation and the duration of the interval during which they had to produce a spoken response. Only when experimenter and participant were sure that the tasks were fully and accurately understood did the participants proceed with the experiment.

Inside the scanner, participants wore earplugs to protect their hearing during scan acquisition and headphones through which they could hear the scanner operator's instructions. Participants could hear their own speech (i.e. responses made during silent 7-s periods between volume acquisitions in the functional MRI scans) attenuated by the earplugs and headphones in addition to feedback via normal bone conduction. Speech was recorded using an MRI-compatible optical microphone (Optoacoustics Optimic 1140, http://www.optoacoustics.com) and the recordings were checked off-line for task compliance. For each of the conditions and the baseline condition, 40 volumes of data were acquired for a total of 120 volumes in each run (18 min); the conditions were intermixed across the 120 volumes in a pseudorandom order so that no condition was presented for more than five trials consecutively. Two runs were acquired in each participant; the two runs had a different pseudorandom order of conditions across the 120 trials and the same order was used for each participant. Stimuli were used only once in the study and were not repeated either within or across the two runs.

\subsection{Speech analysis}

Each participant was recorded reading 80 sentences and describing 80 picture stimuli across the two scan runs, thus they each produced a total of 160 utterances in the scanner. The sentences to be read had an average of 13.8 syllables 
(S.D. $=2.24)$. Sentences were marked as either normal, containing cluttering, containing stuttering, containing both stuttering and cluttering.

\subsection{Image analysis}

The functional images were analysed using the FMRIB Software Library (FSL; http://www.fmrib.ox.ac.uk/fsl; Jenkinson, Beckmann, Behrens, Woolrich, \& Smith, 2012). In addition to the standard motion correction, which generates six motion parameters for inclusion as covariates of no interest in the first-level analysis for each subject, volumes that were motion outliers were included as separate regressors in the model. In three individuals, excessive motion (i.e. $>4 \mathrm{~mm}$, the dimension of a single voxel) was observed during volumes either at the beginning or at the end of a run. These volumes were removed from the time series (i.e. the run was truncated) and the remaining data analysed normally. Each dataset was unwarped using a fieldmap and PRELUDE and FUGUE software running in FSL and spatially smoothed with an 8-mm full-width at half maximum smoothing kernel. A temporal high-pass filter with a cutoff of $150 \mathrm{~s}$ was used to remove low-frequency fluctuations in the signal. Two further regressors were used in the first-level analysis to remove residual image artefacts by extracting the mean time-courses from a $4-\mathrm{mm}$ radius sphere within cerebrospinal fluid of the anterior lateral ventricle (standard space coordinates $2,10,8)$ and white matter in the dorsal posterior frontal lobe $(-26,-22,28)$ (Leech, Braga, \& Sharp, 2012). Images were registered using boundary-based registration (Greve \& Fischl, 2009) to the individual subject's T1-weighted structural image, which in turn was registered using FNIRT (FMRIB's nonlinear registration tool) to the MNI-152 template.

For individual participants, statistical maps were generated to show patterns of activation during each condition relative to baseline and between the Picture Description and the Sentence Reading conditions. The data for the two runs were averaged for each individual participant before group averages and contrasts between groups were analysed using FMRIB's Local Analysis of Mixed Effects stage 1 (Woolrich, Behrens, Beckmann, Jenkinson, \& Smith, 2004). Mixed-effects variance is the sum of fixed-effects variances (estimated within subjects across time at the individual level of analysis) and random effects variance (the between-subjects variances). To summarise the results for each group separately, the data were thresholded at a cluster-forming threshold of $Z>3.1$ and an extent threshold of $p<0.05$ corrected for multiple comparisons. Differences in activation between the two groups did not survive statistical correction for extent (the clusters were not sufficiently large enough) at this threshold, however. We report results for the group differences based on a more liberal uncorrected threshold of $Z>2.3(p<0.01)$ and limit the occurrence of false positives with an additional constraint that cluster size exceeded 30 voxels and, that clusters were located in motor- or language-related brain areas. Group differences at this threshold in occipital cortex or inferior temporal cortex are shown in the figures but are not discussed. Contrast masking was used for the group comparisons to show only regions in which both groups had positive activity $(Z>0)$ for each condition relative to baseline. The purpose of contrast masking was to remove differences between the groups that related to greater de-activations (i.e. higher activity in the baseline condition than in the speaking conditions) in one group compared to the other. Differences in de-activations such as these are difficult to interpret and are most likely false positives.

To evaluate the contribution of a comorbid diagnosis of stuttering to the abnormal patterns of activity seen in the group of adults who clutter, we statistically contrasted two subgroups of adults who clutter and stutter $(N=11)$ and adults who clutter and do not stutter $(N=6)$. Data for these subgroups were compared using FMRIB's Local Analysis of Mixed Effects stage 1 only in regions where the group of adults who clutter showed a functional under- or over-activation relative to controls, namely regions that were located in motor or language related brain areas where the group contrast was significant at $Z>2.3$ $(p<0.01)$, cluster size $>30$ voxels. As for the group contrasts between adults who clutter and control speakers described above, an uncorrected $p<0.01$ threshold was applied for the comparison between these small subgroups.

\section{Results}

\subsection{Speech data}

The adults who clutter rarely exhibited cluttering when speaking inside the scanner; it was observed on average in approximately 6 out of the 160 utterances each participant made (mean $=5.8$ cluttered utterances per participant). The rate of cluttering was observed to occur mainly during the picture description condition (mean $=4.9$ out of 80 sentences) rather than the sentence reading condition (mean $=0.9$ out of 80 sentences).

Within the subgroup of 11 adults who clutter who were also diagnosed with stuttering, 7 exhibited no stuttering within the scanner, which is consistent with their SSI-3 diagnoses of either 'mild' or 'very mild stuttering'. The remaining 4 adults who clutter stuttered on average on less than three of the 160 utterances.

\subsection{Functional imaging data}

The functional MRI data were first analysed by comparing each of the speaking conditions (Picture Description and Sentence Reading) with the baseline for the two groups separately (see Figs. 2 and 3). The patterns of activation in the two groups were then contrasted statistically to reveal between-group differences (see Fig. 4). Finally, the interaction of 


\section{Picture Description > Baseline}
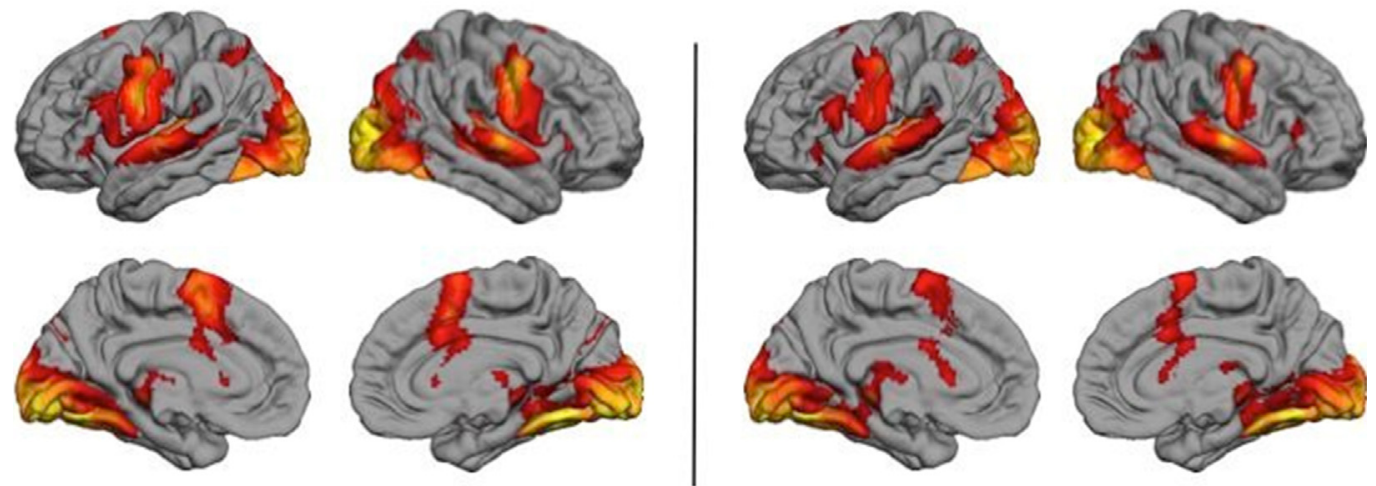

\section{Adults who clutter}

\section{Controls}

Fig. 2. Brain activation during picture description in adults who clutter and controls. Coloured areas represent significantly increased activity during the picture description condition relative to the silent baseline. Statistical images were thresholded using a cluster-forming threshold of $Z>3.1$ and a familywise error threshold on the extent of $p<0.05$, corrected for multiple comparisons. Images were overlaid on the cortical surface using FreeSurfer. Top row: lateral surfaces of the left (left image in each pair) and right hemispheres. Bottom row: medial surfaces of the left (left image in each pair) and right hemispheres. (For interpretation of the references to colour in this figure legend, the reader is referred to the web version of the article.)

condition by group was explored by comparing the two groups on the contrast of Picture Description with Sentence Reading (see Fig. 5).

\subsubsection{Picture description compared to baseline}

During the Picture Description condition, the two groups of adults who clutter and control speakers had very similar patterns of activation (Fig. 2). Activity was significantly increased relative to baseline in the ventral portion of the sensorimotor cortex, superior temporal cortex and occipital cortex bilaterally. The posterior part of the inferior frontal gyrus was activated predominantly on the left (Broca's area). On the medial surface there was extensive activation of the supplementary motor area (SMA and preSMA) extending ventrally to the anterior cingulate cortex. Subcortically, the thalamus was activated extensively and bilaterally as were the anterior lobes of the cerebellum and vermis. In the adults who clutter group, the subcortical activity extended to the striatum (caudate nucleus and putamen) bilaterally.

As can be seen in Fig. 2, the adults who clutter had generally more activity than the control speakers when generating speech for picture description. The statistical comparison of the two groups in this condition did not reveal any significant differences at thresholds corrected for multiple comparisons across the whole brain. Therefore, we used a liberal voxel threshold of $p<0.01(Z>2.3)$ and reported only the regions that had more than 30 voxels above this threshold and were predicted to show differences between these groups, namely the motor and language areas in the brain (see Table 2). At

\section{Sentence Reading > Baseline}

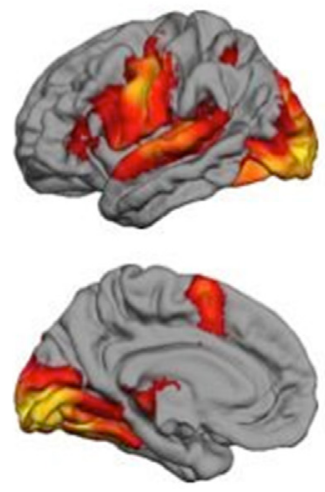

Adults who clutter

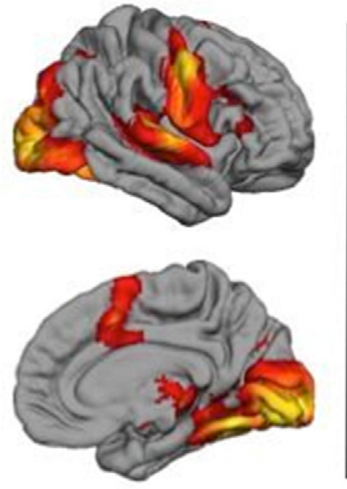

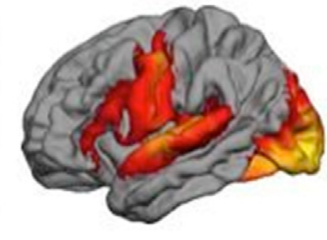
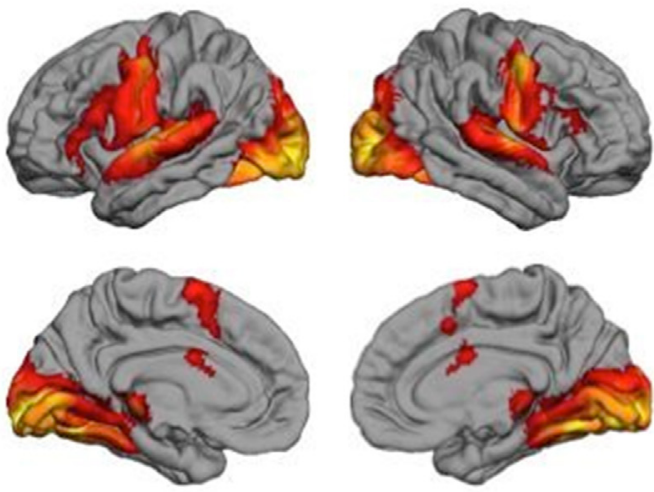

Controls

Fig. 3. Brain activation during sentence reading in adults who clutter and controls. Data are presented as described in the legend to Fig. 2. 


\section{Picture Description > Baseline}

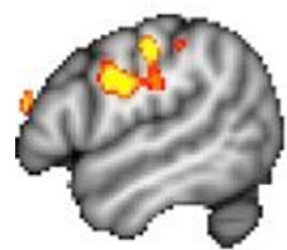

$-52$

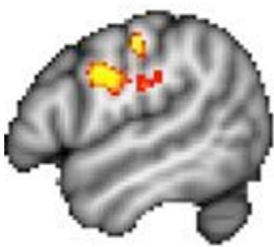

$-52$

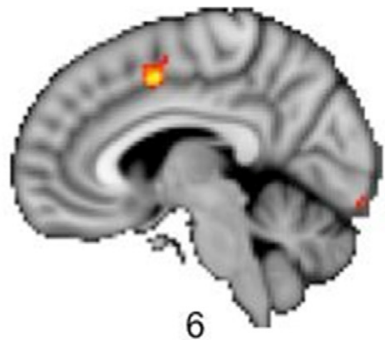

Sentence Reading > Baseline

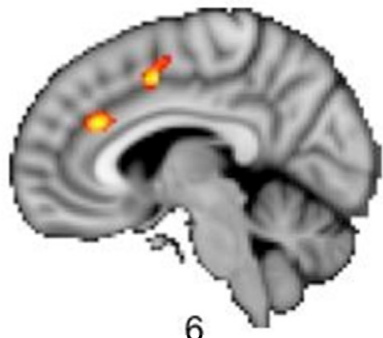

AWC > Controls

Z statistic

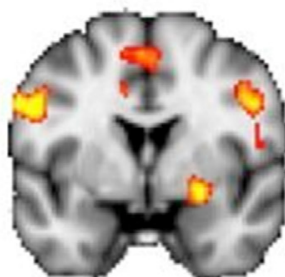

0

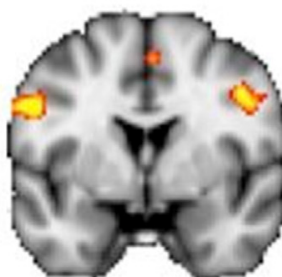

0

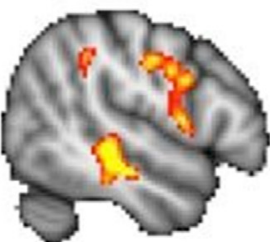

52

Fig. 4. Group differences in activation for the Picture Description (top row) and the Sentence Reading (bottom row) conditions. Coloured statistical maps representing the group comparison are thresholded $(p<0.01, k>30$ voxels, uncorrected) and overlaid on the MNI-152 T1-weighted image. Orange areas show regions where the AWC group had greater activity than the control group. The numbers below each slice are the mm coordinates in MNI-152 standard space for the $y$-axis (coronal slices) and $x$-axis (sagittal slices). The left hemisphere is displayed on the left side of the image. (For interpretation of the references to colour in this figure legend, the reader is referred to the web version of the article.)

Table 2

Regions where there were group differences in activity during Picture Description vs. baseline.

\begin{tabular}{|c|c|c|c|c|c|}
\hline Brain region & Voxels & $Z$ statistic & $x$ & $y$ & $z$ \\
\hline \multicolumn{6}{|l|}{ Adults who clutter $>$ controls } \\
\hline Left preSMA & 614 & 3.62 & -8 & 6 & 50 \\
\hline Right premotor and sensorimotor cortex & 429 & & & & \\
\hline Right frontal operculum & & 3.10 & 52 & 6 & 8 \\
\hline \multirow[t]{3}{*}{ Right ventral premotor cortex } & & 3.10 & 52 & 8 & 28 \\
\hline & & 3.19 & 48 & 2 & 34 \\
\hline & & 3.12 & 52 & 0 & 32 \\
\hline Right postcentral gyrus & & 3.4 & 56 & -8 & 34 \\
\hline Right precentral gyrus & & 3.39 & 54 & -8 & 38 \\
\hline Left premotor and sensorimotor cortex & 800 & & & & \\
\hline Left ventral premotor cortex & & 3.86 & -54 & -4 & 30 \\
\hline \multirow[t]{2}{*}{ Left postcentral gyrus } & & 4.13 & -50 & -18 & 46 \\
\hline & & 3.04 & -50 & -22 & 28 \\
\hline Right putamen & 577 & 3.49 & 28 & -12 & -2 \\
\hline Right superior temporal sulcus & 573 & 3.9 & 54 & -24 & -12 \\
\hline Right angular gyrus/posterior STS & 60 & 2.71 & 58 & -48 & 20 \\
\hline Right posterior temporal gyrus & 148 & 3.25 & 64 & -58 & -16 \\
\hline Left posterior temporal gyrus & 55 & 2.98 & -60 & -66 & -10 \\
\hline \multicolumn{6}{|l|}{ Controls $>$ adults who clutter } \\
\hline Left anterior lobe of the cerebellum & 95 & 2.76 & -44 & -42 & -34 \\
\hline
\end{tabular}

The location of the highest peak in a cluster is given; selected sub-peaks within the large clusters are also described. The number of voxels in a cluster is listed along with the peak height and coordinates of the peak location in MNI-152 standard space. 


\section{Adults who clutter}

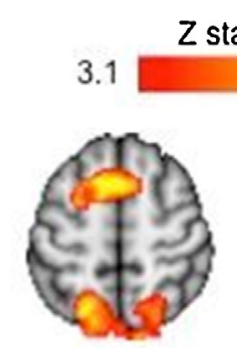

54

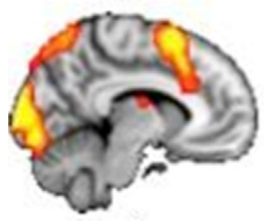

$-8$
5.0

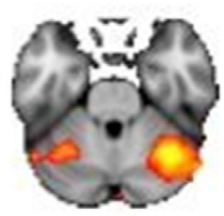

$-28$

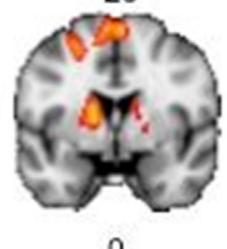

0

\section{Controls}

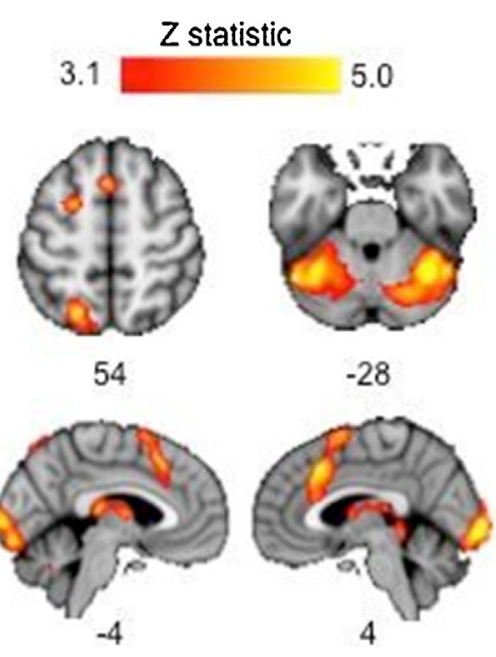

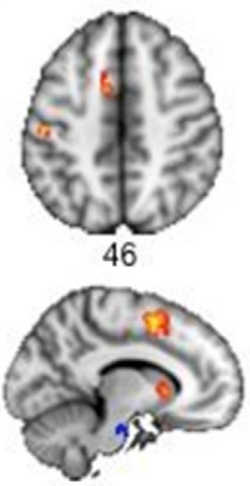

$-10$

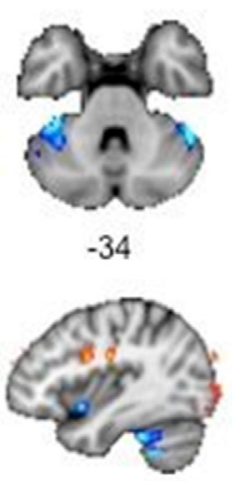

$-38$

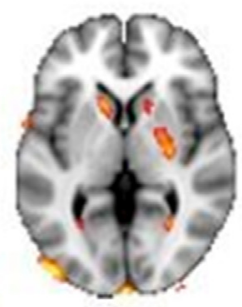

2
AWC > Controls

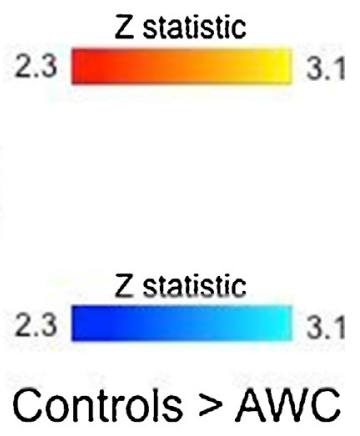

Fig. 5. Brain activation during picture description compared to sentence reading. Top panels: coloured statistical maps for the group averages were thresholded at $Z>3.1, p<0.05$ corrected and overlaid on the MNI-152 T1-weighted image. Orange areas show regions where activity was greater for picture description relative to sentence reading in each group separately. Bottom panel: coloured statistical maps for the comparison between groups were thresholded ( $p<0.01, k>30$ voxels, uncorrected) and overlaid on the MNI-152 T1-weighted image. Orange areas show regions where the AWC group had greater activity than the control group in the picture description > sentence reading contrast. Blue areas show regions where the AWC group had less activity than the control group in the picture description > sentence reading contrast. The numbers below each slice are the mm coordinates in MNI-152 standard space for the $z$-axis (axial slices), $y$-axis (coronal slices) and $x$-axis (sagittal slices). The left hemisphere is displayed on the left side of the image. (For interpretation of the references to colour in this figure legend, the reader is referred to the web version of the article.)

this threshold, there was greater activity in adults who clutter relative to control speakers in the left SMA and preSMA, the sensorimotor cortex and ventral premotor cortex bilaterally, the right posterior superior temporal lobe and right putamen (Fig. 4). There was lower activity in adults who clutter relative to control speakers in the left anterior lobe of the cerebellum.

\subsubsection{Reading compared to baseline}

During the Sentence Reading condition, the two groups had very similar patterns of activation (Fig. 3). Activity was significantly increased relative to baseline in the ventral portion of the sensorimotor cortex, superior temporal and occipital cortex bilaterally. There was also a pattern of left-lateralised activity in the posterior portion of the inferior frontal gyrus. On the medial surface, the SMA (and preSMA) was activated bilaterally extending to the anterior cingulate cortex. Subcortically, there was extensive activity for both groups in the putamen, thalamus and anterior lobes of the cerebellum, bilaterally.

As described above for the picture description condition, during sentence reading, the adults who clutter group had generally more activity than the control speakers group (see Fig. 3). The statistical comparison of the two groups for sentence reading was thresholded as described above for the picture description condition (Table 3 and Fig. 4). At this threshold, there was greater activity in adults who clutter relative to control speakers in the ventral part of the premotor cortex bilaterally, extending posteriorly to the sensorimotor cortex and supramarginal gyrus in the parietal lobe. On the medial surface, there 
Table 3

Regions where there were group differences in activity during Sentence Reading vs. baseline.

\begin{tabular}{|c|c|c|c|c|c|}
\hline Brain region & Voxels & $Z$ statistic & $x$ & $y$ & $z$ \\
\hline \multicolumn{6}{|l|}{ Adults who clutter $>$ controls } \\
\hline Right inferior frontal sulcus & 59 & 2.98 & 52 & 38 & 18 \\
\hline Right anterior cingulate cortex & 283 & 3.22 & 8 & 30 & 26 \\
\hline \multirow[t]{3}{*}{ Right ventral premotor cortex and inferior frontal junction } & 242 & 3.24 & 52 & 8 & 28 \\
\hline & & 3.32 & 46 & 2 & 36 \\
\hline & & 2.75 & 56 & -6 & 38 \\
\hline Right preSMA & 175 & 3.87 & 2 & 6 & 46 \\
\hline Left premotor and sensorimotor cortex & 440 & & & & \\
\hline Left ventral premotor cortex & & 3.73 & -52 & 0 & 30 \\
\hline \multirow[t]{2}{*}{ Left post central gyrus } & & 3.36 & -52 & -16 & 46 \\
\hline & & 2.87 & -50 & -20 & 28 \\
\hline \multirow[t]{2}{*}{ Left supramarginal gyrus } & & 2.71 & -50 & -26 & 32 \\
\hline & & 2.74 & -48 & -34 & 34 \\
\hline Right central sulcus & 50 & 2.89 & 52 & -12 & 58 \\
\hline Right superior temporal sulcus & 454 & 3.78 & 52 & -28 & -6 \\
\hline Right supramarginal gyrus & 68 & 2.91 & 30 & -32 & 36 \\
\hline \multicolumn{6}{|l|}{ Controls $>$ adults who clutter } \\
\hline Left inferior frontal gyrus (pars orbitalis) & 36 & 2.75 & -48 & 20 & -12 \\
\hline
\end{tabular}

The location of the highest peak in a cluster is given; selected sub-peaks within the large clusters are also described. The number of voxels in a cluster is listed along with the peak height and coordinates of the peak location in MNI-152 standard space.

was greater activity in adults who clutter relative to control speakers in the right SMA and preSMA and the anterior cingulate cortex. As for the group comparison of the picture description above, the right superior temporal gyrus was more active in the adults who clutter group than in control speakers. No group differences at this threshold were observed in subcortical areas such as the striatum or cerebellum.

\subsubsection{Picture description versus Reading}

When picture description was compared to sentence reading, greater activity was seen for picture description in several brain regions in both the groups of adults who clutter and control speakers (see Fig. 5). These included extensive portions of the occipital cortex bilaterally extending to the dorsal occipital cortex both medially and laterally. Activity in this cluster also extended to the anterior lobe of the cerebellum bilaterally in both groups. There was also significantly greater activity during picture description relative to sentence reading in the medial frontal cortex bilaterally extending from the preSMA to the anterior cingulate cortex and laterally onto the dorsal surface to include a portion of the left superior frontal sulcus. Both groups also showed significantly more activity during picture description relative to sentence reading in the body of the caudate nucleus bilaterally extending to the anterior and dorsal parts of the thalamus. The adults who clutter but not the control speakers group showed increased activity in the left sensorimotor cortex at about the level of the representation of the face (Fox et al., 2001).

The differences between picture description and sentence reading in the two groups were compared statistically. As for the group contrasts described above for each condition separately, there are no group differences at corrected levels of significance in this contrast of conditions (i.e. the interaction). Results are reported, therefore, at the lower threshold of $p<0.01$ as described above (see Table 4 and Fig. 5). At this threshold the adults who clutter group had more activity than control speakers in the left preSMA, inferior frontal junction, sensorimotor cortex at the level of the face representation, and, subcortically, in the head of the left caudate nucleus and the right putamen (see Fig. 5). The adults who clutter group had less activity than control speakers in the anterior lobe of the cerebellum bilaterally, in the left ventral insula cortex and the left pons (see Fig. 5).

\subsubsection{Subgroup analysis}

To evaluate the contribution of a comorbid diagnosis of stuttering in the group of adults who clutter, we ran a comparison between the 11 adults who clutter and stutter and the 6 adults who clutter and do not stutter. The numbers who were also comorbid for other developmental disorders e.g. dyslexia and dyspraxia were not sufficient to allow further subgroup comparisons but were represented in both subgroups (see Table 1). Only regions where the larger group of adults who clutter $(N=17)$ showed abnormal over- or under-activation relative to control speakers were analysed (this was because it is difficult to interpret differences between these two subgroups that might have occurred in areas that were not functionally abnormal relative to controls). For the Sentence Reading condition only, the adults who clutter and stutter showed greater activity than the subgroup of adults who clutter and do not stutter in the right anterior cingulate cortex $(Z=2.70, x=14$, $y=32, z=24)$ and right ventral premotor cortex $(Z=3.10, x=50, y=4, z=24)$. The percent signal change from these regions is plotted in Fig. 6. There were no differences between these two subgroups in any other areas that were abnormal relative to controls in the Sentence Reading condition or in the Picture Description condition. 
Table 4

Regions where there were group differences in activity during Picture Description vs. Sentence Reading.

\begin{tabular}{|c|c|c|c|c|c|}
\hline Brain region & Voxels & $Z$ statistic & $x$ & $y$ & $z$ \\
\hline \multicolumn{6}{|l|}{ Adults who clutter $>$ controls } \\
\hline Right inferior orbital cortex & 56 & 2.85 & 30 & 26 & -24 \\
\hline Left ventral striatum (caudate nucleus) & 103 & 3.05 & -14 & 18 & -2 \\
\hline Right ventral striatum & 99 & 3.12 & 18 & 16 & -4 \\
\hline Left preSMA & 306 & 3.25 & -14 & 16 & 54 \\
\hline Left inferior frontal junction & 119 & 2.94 & -40 & 2 & 24 \\
\hline Right putamen/globus pallidus & 243 & 2.95 & 30 & -12 & 0 \\
\hline Left sensorimotor cortex (face) & 53 & 3.22 & -36 & -14 & 28 \\
\hline Left postcentral gyrus (ventral) & 48 & 3.02 & -50 & -20 & 46 \\
\hline Left central sulcus (dorsal) & 44 & 2.83 & -28 & -28 & 62 \\
\hline \multicolumn{6}{|l|}{ Controls $>$ adults who clutter } \\
\hline Left ventral anterior insula & 75 & 3.1 & -38 & 6 & -12 \\
\hline Left pons & 38 & 2.6 & -8 & -20 & -30 \\
\hline Right anterior lobe of the cerebellum & 82 & 3.78 & 48 & -44 & -38 \\
\hline Left anterior lobe of the cerebellum & 324 & 3.4 & -40 & -46 & -46 \\
\hline
\end{tabular}

The location of the highest peak in a cluster is given. The number of voxels in a cluster is listed along with the peak height and coordinates of the peak location in MNI-152 standard space.

\section{Discussion}

We scanned a large sample of adults who clutter while they either read sentences that described a visual scene (sentence reading) or they produced their own sentences while viewing a scene (picture description). These conditions were chosen because they placed different demands on the participants in terms of planning an utterance but were well matched for articulatory demands and the final execution of speech. Specifically, we predicted that the picture description condition would place greater demands on linguistic and motor planning compared to the sentence reading condition and that these processes may be dysfunctional in cluttering. This prediction gains support from our observation that the adults who clutter produced more sentences with cluttered speech during picture description than during reading. It should be noted, however, that the production of speech inside the scanner is far from a natural situation. Although, the scanner noise was not present during speech production, it occurred rhythmically (every $9 \mathrm{~s}$ for $2 \mathrm{~s}$ ) and this, coupled with the use of headphones and earplugs, may have had an effect on speech fluency during the study. Below, we first summarise the results of our analysis of the functional MRI data, then we discuss these findings in the context of existing neurobiological models of cluttering and, finally, we discuss the implications of these findings for our current understanding of cluttering.

\subsection{Findings from our functional MRI analysis}

Overall, the two groups of participants (adults who clutter and controls) showed strikingly similar patterns of activation at the cortical level when producing their own speech (for picture description) or when simply reading sentences. Both groups showed greater activity during picture description than during sentence reading in several brain regions including the medial frontal cortex (preSMA and anterior cingulate cortex) bilaterally, left dorsal prefrontal cortex, caudate nucleus and thalamus. This observation is consistent with our prediction that the picture description condition would place greater demands on speech processing than the reading condition and that picture description was a more complex task relative to sentence reading (Picard \& Strick, 1996). It should be noted, however, that although differences were observed in both groups in motor regions, the classic language regions (left inferior frontal and superior temporal cortex) did not show differences for the picture description condition relative to sentence reading in either group.

The differences in activity between the groups at the cortical level reflected, in general, greater activation in the adults who clutter compared to controls. This was especially evident in the premotor cortex on the lateral and medial surfaces and was consistently observed in both the picture description and the sentence reading conditions relative to baseline. Subcortically, however, the group differences were observed in regions, namely the putamen and head of caudate nucleus that were not activated above threshold in controls. We interpret these differences to indicate that, in performing the same task as controls, the group of adults who clutter additionally recruited these subcortical brain regions (though see below for a discussion of interpreting over and underactivity in brain imaging studies). This pattern of subcortical overactivity was evident in the picture description condition relative to baseline, where the right putamen was more active in adults who clutter than in control speakers. For the comparison between the picture description and sentence reading conditions, the right putamen was again more active in adults who clutter than in control speakers but this overactivation of the striatum extended in this contrast to the head of the caudate nucleus bilaterally. Thus, the extra demands required for speech production during picture description relative to sentence reading may be reflected in the recruitment of both the more cognitive (caudate nucleus) and the more motoric (putamen) structures comprising the basal ganglia in adults who clutter.

The pattern of overactivity described above in adults who clutter specifically involved several areas of interest for models of cluttering (see below for discussion). These were the striatum bilaterally (caudate nucleus and putamen), the left preSMA 


\section{Right ventral premotor cortex}

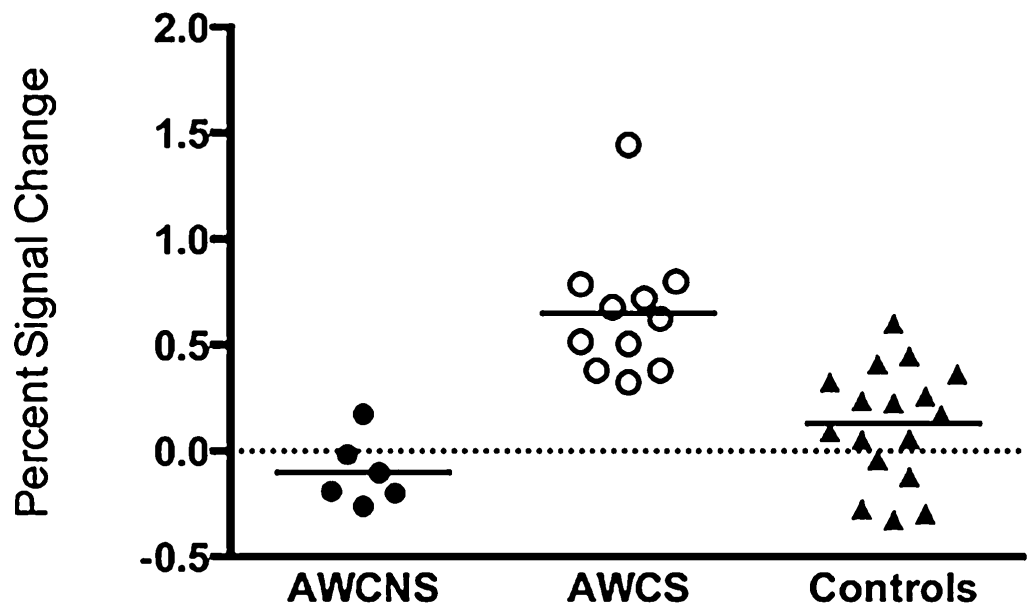

Right anterior cingulate cortex

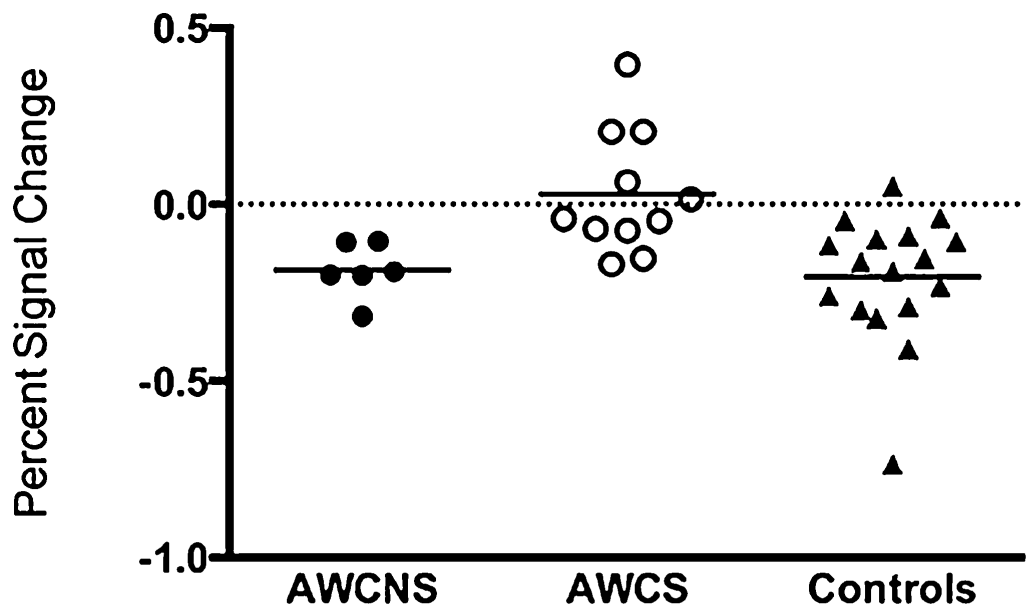

Fig. 6. Differences in brain activity during Sentence Reading in AWC related to a comorbid diagnosis of stuttering. Percent signal change from the Sentence Reading condition relative to baseline is plotted for individual subjects. Filled circles, people who clutter and do not stutter (AWCNS); open circles, people who clutter and stutter (AWCS); triangles, controls. Line, group mean.

and anterior cingulate cortex (ACC) on the medial wall, and several regions in the lateral premotor cortex including portions of pre- and post-central gyri, two of which were located at the level of the representation of the face (Fox et al., 2001). The striatum receives projections from most of the cortex. The caudate nucleus, in particular, gets input from the prefrontal cortex, whereas the putamen is a target of sensorimotor cortex (located on the pre- and post-central gyri). The preSMA is a target for outputs from the basal ganglia via the thalamus (Picard \& Strick, 1996). Our results are consistent with the notion of dysfunction in these cortico-striatal-thalamo-cortico loops in cluttering, therefore. Similar dysfunction in loops through the basal ganglia are thought to play a role in disorders of motor control such as Parkinson's disease, Tourette's syndrome and dystonia.

The group of adults who clutter showed reduced activity relative to controls in only one brain region: the anterior lobe of the cerebellum. This region was extensively activated bilaterally in both controls and adults who clutter during picture description relative to sentence reading but there was significantly less activity in the group of adults who clutter relative to the control group in the lateral portions of the anterior cerebellar lobe (particularly on the right side). The location of cerebellar areas showing reduced activation in adults who clutter corresponds to lobule V1. A recent meta-analysis of 
cerebellar function identified consistent language-related activation in lobule VI of the cerebellum particularly on the right for people who were left lateralised for language (Stoodley \& Schmahmann, 2009); the anterior lobe was noted to show involvement in particular for tasks involving articulation (Fiez \& Raichle, 1997). Lobule VI is functionally connected with cortical areas in the inferior frontal gyrus, SMA and ACC that were overactive in the group of adults who clutter (Buckner, Krienen, Castellanos, Diaz, \& Yeo, 2011). The relationship between these overactive cortical areas and underactive cerebellum in adults who clutter is as yet unclear.

Thus far, the brain areas described as abnormally active in adults who clutter have been in regions associated with motor planning (preSMA), motor execution (SMA, premotor and sensorimotor cortex and putamen, cerebellum), linguistic selection and cognitive control (caudate nucleus and ACC). There was additional overactivity in adults who clutter in two areas outside this network, namely, the right superior temporal sulcus and right angular gyrus. Activity in these regions is not strongly associated with language processing, though they are commonly activated in the left hemisphere during multisensory speech perception and in reading.

\subsection{Interpreting differences in brain activity between groups}

Before discussing these results further, it is worth considering the interpretation of differences between groups in the amount of activity indexed by relative increases or decreases in the height and extent of the functional MRI signal. There are many potential explanations for these differences. One possibility is that differences between the two groups in speech performance - such as syllable rate, sentence durations, amount of speech produced - would affect the amount of brain activity observed. Even though such effects have been reported previously using other brain imaging methods, such as positron emission tomography in which activity is averaged over about a minute (Sidtis, Strother, \& Rottenberg, 2003), we think these effects are unlikely to explain the differences seen in our study. In the current study, we used sparse-sampling functional MRI, in which a whole brain volume is acquired at the peak of the haemodynamic response to an event occurring approximately five seconds earlier. The timing of our acquisition was intended to capture the brain's response to speech production occurring at 1-3s into the sentence reading or picture description. Subsequent continued speech would not affect this response, therefore. It is possible that speech rate during this window of speech production may have affected the signal but we think the influence would be small and highly variable, given that only the peak of the response is measured. Furthermore, our preliminary analysis of the speech recordings made from inside the scanner does not reveal reliable differences between the groups in speech production.

Further explanations for differences in the amount of activity in adults who clutter relative to controls include the idea that adults who clutter may be engaged in different processes to achieve the same task as controls, and may do so within different time frames. In addition, there may be unknown differences in physiological mechanisms between the two groups that underpin these differences in activation levels in the network of brain areas recruited to perform a task. Often, when discussing "overactivity" or "underactivity" in one group relative to another, explanations relating to efficiency of processing are invoked; the idea being that more practiced and expert task performance is related to more efficient neural processing resulting in less activity. Such an interpretation is tempting when discussing brain activity in developmental disorders of speech such as stuttering and cluttering as they are consistent with the idea that speech is produced with more effort and less smoothly by individuals with these difficulties. It has been argued recently, however, that such explanations are not useful and simply redescribe the data (Poldrack, 2014). Until our understanding of these effects improves, we are limited to saying that the two groups differ in the degree to which brain areas are activated when performing a task, which suggests that the processing is abnormal in adults who clutter, defined as being different to that seen in a control population.

\subsection{Findings within the context of existing speculation on the neurological underpinnings of cluttering}

Our findings of abnormal activation in adults who clutter are strikingly in agreement with predictions from Alm's (2011) theoretical framework regarding the neurological basis of cluttering, specifically the claim that "the core of the problems in cluttering is located in the medial wall of the left frontal lobe" $(2011$, p. 4). This proposal was based on the known roles played by the medial cortex in mediating and coordinating spontaneous speech, and in motor speech execution and speech monitoring. Key brain areas in this model in include the ACC, preSMA, SMA and basal ganglia outputs to this region. According to this model, these structures comprise an "executive hub" that collects linguistic information from linguistic centres in the left lateral cortex, such as Broca's and Wernicke's areas, assembles words and sequences phrases. The precise control of articulation, in particular of timing and speech rate (critical to a core definition of cluttering) would be under the control of a circuit including the SMA, cerebellum and basal ganglia (note that Seeman originally implicated the basal ganglia dysfunction as contributory to cluttering). Selection of individual words and suppression of competitors might also be mediated by the basal ganglia. Monitoring of the auditory consequences of speech production would also involve the ACC and SMA. Furthermore, Alm (2011) specifically suggests that cluttering might be characterised by dysregulation and hyperactivation of the medial frontal cortex, which could be secondary to disinhibition of the basal ganglia output, possibly related to a hyperactive dopaminergic system. The conclusion is that cluttering can be explained by dysregulation of the ACC/SMA circuitry, and that should the disorder eventually be seen specifically as a speech rate and motor speech planning disorder, then the SMA alone is likely to be heavily involved. 
In the current study, the group of adults who clutter demonstrated both hyperactivity of the medial frontal cortex and dysregulation of the basal ganglia. But, our findings point to the dysfunction in the medial wall being centred on the preSMA in adults who clutter extending to encompass the very closely neighbouring cortex of the ACC. The more posteriorly located SMA was not abnormally activated alone in adults who clutter, however, and there was greater involvement of lateral premotor and prefrontal cortex than predicted by Alm's model.

It is worth noting that dysfunction in this network of areas involved in speech motor control was observed during both speech tasks and against a background of relatively fluent speech production, with little occurrence of cluttering. For these reasons, the findings feel like clear evidence of trait rather than state differences in cluttering.

\subsection{Implications for a working model of the neural underpinnings of cluttering}

Findings, both at cortical and subcortical levels point to speech motor involvement in cluttering, with abnormalities detected predominantly in motor regions. Despite contentions that cluttering also involves impairment in linguistic planning, we find no clear evidence for abnormal function in adults who clutter in higher-order language areas, other than a region of the left inferior frontal gyrus that is slightly more dorsally located than the cortical area traditionally called "Broca's area". Nevertheless, it is striking that we found greater impairment both behaviourally (slightly more cluttering) and in terms of brain activity in the picture description condition than in the reading condition. It could be argued that the picture description task requires greater linguistic demands than reading but note that this did not manifest itself in greater activity in classic "language" areas. However, other functions such as attention and short-term memory are also differentially involved between the sentence reading and picture description conditions. Also, even though both conditions compared to baseline showed increased activation in adults who clutter of right hemisphere homologues of posterior "language" regions (superior temporal sulcus and angular gyrus), these regions were not differentially activated for picture description relative to sentence reading. The immediate implications of the findings observed in these two conditions in these posterior language regions are not immediately clear, but the findings are of note because some researchers have suggested a relationship between cluttering and high level language comprehension difficulties (Daly, 1986; Ward, 2006; Weiss, 1964).

The anterior cingulate cortex is an extensive band of tissue that sits above the corpus callosum on the medial wall of each hemisphere. It comprises a heterogeneous set of cortical regions that differ in cytoarchitecture and functional roles. In our study, the extensive overactivation of the neighbouring preSMA in adults who clutter encompassed parts of the subjacent ACC. In the reading condition, a more anterior portion of the ACC in the right hemisphere was overactive in adults who clutter. This region is close to the location of the cingulate motor area, which has direct connections with regions involved in vocalisation including periaqueductal grey, premotor and motor cortex and the preSMA (see Paus, 2001 for a review). It should also be noted that activity in the ACC has been associated with cognitive control, error detection and inhibition (see below).

\subsection{Cluttering and stuttering}

A complicating factor in most studies of cluttering is that it rarely occurs in isolation (St. Louis et al., 2007). Stuttering is the most common and significant comorbid diagnosis (33-70\%; see Ward, 2006) that occurs with cluttering (St. Louis et al., 2007; Van Zaalen, Wijnen, \& De Jonckere, 2009a; Ward, 2010).

The relationship between cluttering and stuttering is in one sense counterintuitive: stuttering is characterised by a slower speech rate, physical difficulties with articulation, associated state anxiety, avoidance (of linguistic and environmental variables), and struggle in maintaining fluent speech. The core behaviours of cluttering (as defined by St. Louis \& Schulte, 2011) might almost seem as the opposite, with many adults who clutter presenting with what could be described as 'hyperfluent' speech. Furthermore, although intelligibility frequently suffers in cluttering, there may well be no speech-related anxiety and often a marked lack of awareness of any difficulty, and lack of concern (Daly \& Cantrell, 2006; Ward, 2006).

Consistent with these previous reports, the group of adults who clutter in the current study were heterogeneous with respect to comorbid diagnoses, with a large single subgroup comprising 11 adults who clutter and stutter. We attempted to disentangle the contributions that a comorbid diagnosis of stuttering might have on the results described by comparing the two subgroups of adults who clutter and stutter and those who clutter but did not stutter. Only two of the regions described as functionally abnormal in adults who clutter relative to controls showed any differences due to stuttering: there was greater activity in the right ventral premotor cortex and right ACC in the subgroup of adults who clutter and stutter compared with those who clutter but do not stutter. Both these regions have been previously linked with inhibitory control and error-related activity (e.g. Xue, Aron, \& Poldrack, 2008). The lateral premotor cortex is also important for auditory feedback (Guenther, Ghosh, \& Tourville, 2006). A plausible explanation of the overactivity seen in these regions in adults who clutter and stutter but lower levels of activity in adults who clutter and do not stutter might relate to control and awareness of speech errors associated with stuttering. Also, people who stutter tend to show anxiety in addition to awareness of their speech errors. Future studies should implement the use of measures of state and trait anxiety to address the influence of such effects on brain imaging data of such populations. The effect of a diagnosis of stuttering was not observed to significantly affect any of the other regions that were abnormally activated in adults who clutter compared to controls. Nevertheless, further work will compare another group of adults who stutter with the adults who clutter and controls described here to further understand the similarities and differences in patterns of brain activity associated with these disorders. 
In addition to the comorbid diagnosis of stuttering, there were smaller numbers of individuals in the adults who clutter who also were dyspraxic or dyslexic. Due to these small numbers it was not possible to determine the contribution that these other developmental disorders made to the results reported here. We can speculate, however, that this contribution is likely to be small due to the large sample size and the relatively small numbers with other diagnoses. Overall, the heterogeneity of the adults who clutter group has most likely resulted in reduced sensitivity of finding significant group differences, i.e. the possibility of false negative results is increased.

\section{Conclusions}

Our findings showed that adults who clutter had greater activity in the premotor cortex on the lateral surface bilaterally and on the medial surface (preSMA) compared with a matched control group. Adults who clutter also showed increased activity in basal ganglia structures and reduced activity in the lateral anterior cerebellum bilaterally in comparison with controls. The pattern of abnormal brain activity was highly consistent with existing theory on the neurology of cluttering, and reflects problems with motor control across planning and execution levels.

\section{CONTINUING EDUCATION}

\section{MQCS}

QUESTIONS

1. Regarding concepts in the introduction:

(a) St. Louis and Schulte's (2011) LCD definition includes language deficits as one of the core characteristics of the disorder

(b) Ward (2006) argues that cluttering is a language based disorder

(c) Alm's (2011) executive hub model contends that cluttering is associated with deficits with cerebellar circuitry

(d) Cluttering rarely co-occurs with stuttering

(e) Ward (2011a) contends that cluttering is represented at all four levels of van der Merwe's (2008) model of motor speech processing

2. Regarding participants:

(a) All AWC had co-occurring communication disorders

(b) All AWC were diagnosed using a the cluttering spectrum definition

(c) Cluttering was diagnosed in the AWC group using the St. Louis and Schulte LCD definition criteria

(d) In the AWCS subgroup, stuttering severity ranged from mild to moderate as measured by SSI-3

(e) Only right handed participants were selected for this study

3. Regarding methodology:

(a) Data were recorded under 4 conditions in the scanner; oral reading, picture description, responding to verbally presented questions and (silent) baseline

(b) Forty volumes of data were recorded for a total of 160 volumes in each of the two runs

(c) A sparse sampling method of data collection was used

(d) Participants were encouraged to speak in the baseline condition if they had not finished their sentence from the previous picture description or oral reading task

(e) Participants were required to listen to ambient music during baseline recordings

4. Regarding findings:

(a) AWC showed reduced cerebellar activation for spontaneous speech

(b) AWC showed a decrease in activity in Broca's area in both spontaneous speech and oral reading conditions

(c) AWCNS subgroup showed increased activity in the putamen over the AWCS group in the spontaneous speech task

(d) AWC showed increased preSMA activity over CON in the spontaneous speech condition

(e) Differences between AWC and CON were seen only at a subcortical level

5. Regarding interpretation of results:

(a) Cluttering is associated with anomalous motor speech control at programming and planning levels.

(b) Cluttering and stuttering are viewed as having the same neural underpinnings

(c) Findings support the notion that cluttering is a language based disorder

(d) Cluttering is associated with motor speech processing problems specifically at the motor execution/articulation level

(e) There is no support for the idea that cluttering can be associated with difficulties with the inhibition of competing motor commands

\section{Acknowledgments}

This work was supported by a Stammer Trust grant and a Reading University Functional Imaging Facility New Directions grant, both awarded to Dr. Ward. Emily Connally was funded by a Clarendon OUP Fund scholarship. 


\section{Financial disclosures}

Christos Pilatsikis and Kate Watkins did not report other relevant financial relationships used in support of the research reported in this article.

\section{Non-financial disclosures}

David Ward, Emily Connally, Christos Pilatsikis and Kate Watkins did not disclose any relevant non-financial relationships used in support of the research reported in this article.

\section{References}

Alm, P. A. (2010). The dual premotor model of cluttering and stuttering: A neurological framework. In K. Bakker, L. Raphael, \& F. Myers (Eds.), Proceedings of the First World Conference on Cluttering Katarino, Bulgaria, 2007, (pp. 207-210). http://associations.missouristate.edu/ICA

Alm, P. (2011). The nature and neurology of cluttering. In D. Ward, \& K. Scaler Scott (Eds.), Cluttering: A handbook of research, intervention and education (pp. 3-28). Hove: Psychology Press.

Buckner, R. L., Krienen, F. M., Castellanos, A., Diaz, J. C., \& Yeo, B. T. T. (2011). The organization of the human cerebellum estimated by intrinsic functional connectivity. Journal of Neurophysiology, 106(5), 2322-2345.

Daly, D. A. (1986). The clutterer. In K. O. St. Louis (Ed.), The atypical stutterer (pp. 155-192). New York: Academic Press.

Daly, D. A., \& Cantrell, R. P. (2006, July 27). Cluttering characteristics identified as diagnostically significant by 60 fluency experts. In Paper presented at the International Fluency Congress Dublin, Ireland,

Fiez, J., \& Raichle, M. (1997). Linguistic processing. In J. Schmahmann (Ed.), The cerebellum and cognition (pp. 233-254). San Diego: Academic Press.

Fox, P. T., Huang, A., Parsons, L. M., Xiong, J. H., Zamarippa, F., Rainey, L., et al. (2001). Location-probability profiles for the mouth region of human primary motor-sensory cortex: Model and validation. Neuroimage, 13(1), 196-209.

Greve, D. N., \& Fischl, B. (2009). Accurate and robust brain image alignment using boundary-based registration. Neuroimage, 48(1), 63-72.

Guenther, F. H., Ghosh, S. S., \& Tourville, J. A. (2006). Neural modeling and imaging of the cortical interactions underlying syllable production. Brain and Language, 96, 280-301.

Jenkinson, M., Beckmann, C. F., Behrens, T. E., Woolrich, M., \& Smith, S. M. (2012). FSL. Neuroimage, 62, 782-790.

Leech, R., Braga, R., \& Sharp, D. J. (2012). Echoes of the brain within the posterior cingulate cortex. Journal of Neuroscience, 32(1), 215-222.

Lebrun, Y. (1996). Cluttering after brain damage. Journal of Fluency Disorders, 21, 289-295.

Paus, T. (2001). Primate anterior cingulate cortex: Where motor control, drive and cognition interface. Nature Reviews Neuroscience, 2, 417-424.

Picard, N., \& Strick, P. L. (1996). Motor areas of the medial wall: A review of their location and functional activation. Cerebral Cortex, 6, 342-353.

Poldrack, R. A. (2014). Is "efficiency" a useful concept in cognitive neuroscience? Developmental Cognitive Neuroscience, http://dx.doi.org/10.1016/j. dcn.2014.06.001 [Epub ahead of print]

Preus, A. (1996). Cluttering upgraded. Journal of Fluency Disorders, 21, 349-358.

Riley, G. D. (1994). Stuttering severity instrument for children and adults (SSI-3). Austin, TX: Pro Ed.

Seeman, M. (1970). Relations between motorics of speech and general motor ability in clutterers. Folia Phoniatrica, 22, 376-380.

Sidtis, J. J., Strother, S. C., \& Rottenberg, D. A. (2003). Predicting performance from functional imaging data: Methods matter. Neuroimage, $20,615-624$.

St. Louis, K. O., Myers, F. L., Bakker, K., \& Raphael, L. J. (2007). Understanding and treating cluttering. In E. Conture, \& R. Curlee (Eds.), Stuttering and related disorders of fluency (pp. 297-325). New York: Thieme.

St. Louis, K. O., \& Schulte, K. (2011). Defining cluttering: The lowest common denominator. In D. Ward, \& K. Scaler Scott (Eds.), Cluttering: a handbook of research, intervention and education (pp. 233-253). Hove: Psychology Press.

Stoodley, C. J., \& Schmahmann, J. D. (2009). Functional topography in the human cerebellum: A meta-analysis of neuroimaging studies. Neuroimage, 44(2), 489-501.

Van der Merwe, A. (2008). A theoretical framework for the characterization of pathological speech sensorimotor control. In M. R. McNeil (Ed.), Clinical management of sensorimotor speech disorders (pp. 3-18). Stuttgart: Thieme.

Van Riper, C. (1982). The nature of stuttering. Englewood Cliffs: Prentice-Hall.

Van Zaalen, Y., Wijnen, F., \& De Jonckere, P. (2009a). Differential diagnostic characteristics between cluttering and stuttering-Part one. Journal of Fluency Disorders, 34, 137-154.

Van Zaalen, Y., Wijnen, F., \& De Jonckere, P. (2009b). Language planning disturbances in children who clutter or have learning disabilities. International Journal of Speech and Language Pathology, 11, 496-508.

Ward, D. (2006). Stuttering and cluttering: Frameworks for understanding and treatment. Hove: Psychology Press.

Ward, D. (2010). Stuttering and normal nonfluency: Cluttering spectrum behaviour as a functional descriptor of abnormal fluency. In K. Bakker, L. Raphael, \& F. Myers (Eds.), Proceedings of the First World Conference on Cluttering Katarino, Bulgaria, (pp. 261-267). http://associations.missouristate.edu/ICA

Ward, D. (2011a). Motor speech control and cluttering. In D. Ward, \& K. Scaler Scott (Eds.), Cluttering: A handbook of research, intervention and education (pp. 34-44). Hove: Psychology Press.

Ward, D. (2011b). Scope and constraint in the diagnosis off cluttering: Combining two perspectives. In D. Ward, \& K. Scaler Scott (Eds.), Cluttering: A handbook of research, intervention and education (pp. 254-262). Hove: Psychology Press.

Weiss, D. A. (1964). Cluttering. Englewood Cliffs, NJ: Prentice-Hall.

Woolrich, M. W., Behrens, T. E., Beckmann, C. F., Jenkinson, M., \& Smith, S. M. (2004). Multilevel linear modelling for FMRI group analysis using Bayesian inference. Neuroimage, 21, 1732-1747.

Xue, G., Aron, A. R., \& Poldrack, R. A. (2008). Common neural substrates for inhibition of spoken and manual responses. Cerebral Cortex, 18(8), $1923-1932$.

David Ward is a lecturer and researcher at the University of Reading. He is author of a textbook, 'stuttering and cluttering' (2006), and more recently co-editor of a volume on cluttering (2011) and a manual on the practical management of cluttering (2013). A qualified speech language therapist, he maintains a specialist clinical practice at the Apple House centre for stammering in Oxford, UK.

Emily Connally is pursuing a DPhil in the Department of Experimental Psychology at the University of Oxford under the supervision of Prof. Kate Watkins. Her thesis is on the neurobiological underpinnings of persistent developmental stuttering and primarily focuses on the function and integration of the speech and motor networks. Previously, she studied healthy ageing processes at Harvard University, and thereafter at the Massachusetts Institute of Technology with a focus on emotional memory and early cognitive markers of Parkinsonism.

Christos Pliatsikas did his PhD in the Department of Clinical Language Sciences, University of Reading in November 2010, and went on to work as a postdoctoral Research Assistant at the Centre for Integrative Neuroscience and Neurodynamics (CINN) until June 2011. After working as a Research 
Fellow at the School of Psychology, University of Birmingham, he returned to the CINN between March 2012 and April 2013 in the role of the MRI Operations officer. He currently is a lecturer in Cognitive Psychology at the University of Kent.

Jess Bretherton-Furness completed her BSc degree in speech and language therapy at The University of Reading. Since then she has combined clinical posts in Kent and Berkshire (UK) with research into possible high level language disturbances among those who clutter. She is currently studying for a PhD at The University of Reading under Dr Ward's supervision. Her Doctoral thesis extends previous work into language processing in people who clutter and stutter.

Kate Watkins is a University Professor in Experimental Psychology and Fellow of St. Anne's College at the University of Oxford. She trained in neuropsychology and brain imaging at University College London and the Montreal Neurological institute in Canada. She uses brain imaging to study the neural basis of genetic, developmental and acquired disorders affecting speech and language. 